\title{
Modified fragmentation function in heavy ion collisions at RHIC via direct $\gamma$-jet measurements
}

\author{
A.M. Hamed ${ }^{\mathrm{a}}$ \\ for the STAR Collaboration \\ Physics Department, Cyclotron Institute, Texas A\&M University, College Station, TX 77843, USA
}

Received: 8 September 2008 / Revised: 9 December 2008 / Published online: 25 February 2009

(C) Springer-Verlag / Società Italiana di Fisica 2009

\begin{abstract}
The presented results are the first measurements at RHIC for direct $\gamma$-charged hadron azimuthal correlations in heavy ion collisions. We use these correlations to study the color charge density of the medium through the mediuminduced modification of high- $p_{T}$ parton fragmentation. Azimuthal correlations of direct photons at high transverse energy $\left(8<p_{T}<16 \mathrm{GeV}\right)$ with away-side charged hadrons of transverse momentum $\left(3<p_{T}<6 \mathrm{GeV} / c\right)$ have been measured over a broad range of centrality for $\mathrm{Au}+\mathrm{Au}$ collisions and $p+p$ collisions at $\sqrt{s_{N N}}=200 \mathrm{GeV}$ in the STAR experiment. A transverse shower shape analysis in the STAR Barrel Electromagnetic Calorimeter Shower Maximum Detector is used to discriminate between the direct photons and photons from the decays of high $p_{T} \pi^{0}$. The per-trigger away-side yield of direct $\gamma$ is smaller than from $\pi^{0}$ trigger at the same centrality class. Within the current uncertainty the $I_{C P}$ of direct $\gamma$ and $\pi^{0}$ are similar.
\end{abstract}

PACS 25.75.Bh $\cdot 25.75 . \mathrm{Cj}$

\section{Introduction}

Despite the lack of understanding of the early time dynamics, including the modifications of the accelerated nuclei even before the collisions, various complementary measurements from RHIC data have revealed the formation of a strongly coupled medium in contrast to the expected weakly coupled medium [1-8]. Determining the characteristics of the formed medium is the main goal for the current RHIC heavy ion program. One of the most important of such characteristics is the medium color charge density, which might lead to understand the medium dynamics. Actually, the color structure of the medium can be probed by its effect on the

a e-mail: ahamed@ tamu.edu propagation of a fast parton [9]. The characterization of the resulting medium-induced modification of high- $p_{T}$ parton fragmentation has become one of the most active areas of research stimulated by RHIC data at the experimental and theoretical levels.

The first three years of RHIC have produced one of the most celebrated results at RHIC which is the suppression of hadrons in central $\mathrm{Au}+\mathrm{Au}$ collisions, compared to $p+p$ collisions [10] and to cold nuclear matter [11] at high $p_{T}$. This suppression is well described by very different pQCDbased energy loss models in the light flavor sector [12]. However, the observation of a similar level of suppression for the heavy flavor [13], and the unexpected smaller suppression of baryons compared to mesons at intermediate to high $p_{T}$ [14-16], cannot be easily reconciled in these pQCD-based energy loss models that incorporate gluon radiation as the main source of in-medium energy loss. In fact the applicability of pQCD in describing the parton-matter interaction has been increasingly challenged by the strongly coupled nature of the produced matter at RHIC. As a result there is no single commonly accepted calculation of the underlying physics to describe in-medium energy loss for different quark generations as well as for the gluon.

Experimental observables based on single-particle spectra are not sensitive enough to discriminate between the different energy loss mechanisms [17], indicating the need for additional experimental observables in order to better constrain the energy loss mechanism. While the initial parton energy is not accessible in single-particle spectra, a better sensitivity to the initial parton energy is obtained by the dihadron azimuthal correlation measurement, and therefore it is expected to provide a slightly better way in constraining the energy loss. Model-dependent detailed studies [18] have shown that the azimuthal correlation measurements of dihadrons are more sensitive than single particle spectra but both have diminished sensitivity at high gluon density due to the geometrical bias. The $\gamma$-hadron azimuthal correlations 
measurement provides a powerful way to quantify the energy loss through the dominant process QCD Compton-like scattering since the photon transverse momentum balances the parton initial energy [19], modulo negligible corrections from initial state radiation. In particular the $\gamma$-hadron azimuthal correlations provide a unique way to quantify the energy loss dependence on the initial parton energy and possibly the color-factor.

\section{Data analysis}

Using a level-2 high- $p_{T}$ tower trigger to tag $\gamma$-jet events, in 2007 the STAR experiment collected an integrated luminosity of $535 \mu \mathrm{b}^{-1}$ of $\mathrm{Au}+\mathrm{Au}$ collisions at $\sqrt{s_{N N}}=$ $200 \mathrm{GeV}$. The level-2 trigger algorithm was implemented in the Barrel Electromagnetic Calorimeter (BEMC) and optimized based on the information of the direct $\gamma / \pi^{0}$ ratio in $\mathrm{Au}+\mathrm{Au}$ collisions [20], the $\pi^{0}$ decay kinematics, and the electromagnetic shower profile characteristics. The BEMC has a full azimuthal coverage and pseudorapidity coverage $|\eta| \leq 1.0$. As a reference measurement we use $p+p$ data at $\sqrt{s_{N N}}=200 \mathrm{GeV}$ taken in 2006 with integrated luminosity of $11 \mathrm{pb}^{-1}$. The Time Projection Chamber (TPC) was used to detect charged particle tracks and measure their momenta. The charged track quality cuts are similar to previous STAR analyses [21]. For this analysis, events with at least one cluster with $E_{T}>8 \mathrm{GeV}$ were selected. To ensure the purity of the photon triggered sample trigger towers were rejected if a track with $p>3 \mathrm{GeV} / c$ points to it.

A crucial step of the analysis is to discriminate between showers of direct $\gamma$ and two close $\gamma$ 's from a high- $p_{T} \pi^{0}$ symmetric decay. At $p_{T} \sim 8 \mathrm{GeV} / c$ the angular separation between the two photons resulting from a $\pi^{0}$ symmetric decay (both decays photons have similar energy, smallest opening angle) at the BEMC face is typically smaller than the tower size $(\Delta \eta=0.05, \Delta \phi=0.05)$; but a $\pi^{0}$ shower is generally broader than a single $\gamma$ shower. The Barrel Shower Maximum Detector (BSMD), which resides at $\sim 5 \mathrm{X}_{0}$ inside the calorimeter towers, is well-suited for $(2 \gamma) /(1 \gamma)$ separation up to $p_{T} \sim 26 \mathrm{GeV} / c$ due to its fine segmentation $(\Delta \eta \approx 0.007, \Delta \phi \approx 0.007)$. In this analysis the $\pi^{0} / \gamma$ discrimination was carried out by making cuts on the shower shape as measured by the BSMD, where the $\pi^{0}$ identification cut is adjusted in order to obtain very pure sample of $\pi^{0}$ and a sample rich in direct $\gamma\left(\gamma_{\text {rich }}\right)$. The discrimination cuts are varied to determine the systematic uncertainties. To determine the combinatorial background level the relative azimuthal angular distribution of the associated particles with respect to the trigger particle per trigger is fitted with a two Gaussian peaks and a straight line. The near- and away-side yields, $Y^{n}$ and $Y^{a}$, of associated particles per trigger are extracted by integrating the $1 / N_{\text {trig }} d N / d(\Delta \phi)$ distributions in $|\Delta \phi| \leq 0.63$ and $\Delta \phi-\pi \leq 0.63$ respectively. The yield is corrected for the tracking efficiency of associated charged particles as a function of multiplicity.

The shower shape cuts used to select a sample of directphoton-"rich" triggers reject most of the $\pi^{0}$ 's, but do not reject photons from highly asymmetric $\pi^{0}$ decays, $\eta$ 's, and fragmentation photons. All of these sources of background are removed as follows (Eq. 2.1), but only within the systematic uncertainty resulting from the assumption that their correlations are similar to those for $\pi^{0}$ 's. Assuming zero nearside yield for direct photon triggers and a very pure sample of $\pi^{0}$, the away-side yield of hadrons correlated with the direct photon is extracted as

$Y_{\gamma_{\text {direct }}+h}=\frac{Y_{\gamma_{\text {rich }}+h}^{a}-R Y_{\pi^{0}+h}^{a}}{1-R}, \quad R=\frac{Y_{\gamma_{\text {rich }}+h}^{n}}{Y_{\pi^{0}+h}^{n}}$.

Where $Y_{\gamma_{\text {rich }}+h}^{a(n)}$ and $Y_{\pi^{0}+h}^{a(n)}$ are the away (near)-side yields of associated particles per $\gamma_{\text {rich }}$ and $\pi^{0}$ triggers respectively, so that $R$ is the fraction of $\gamma_{\text {rich }}$ triggers that are actually from $\pi^{0}, \eta$, and fragmentation photons.

\section{Results}

Figure 3.1 (left) shows the azimuthal correlation for inclusive photon triggers for the most peripheral and central bins in $\mathrm{Au}+\mathrm{Au}$ collisions. Parton energy loss in the medium causes the away-side to be increasingly suppressed with centrality as it was previously reported $[11,21]$. The suppression of the near-side yield with centrality, which has not been observed in the charged hadron azimuthal correlation, is consistent with an increase of the $\gamma / \pi^{0}$ ratio with centrality at high $E_{T}^{\text {trig }}$. The shower shape analysis is used to distinguish between the $(2 \gamma) /(1 \gamma)$ showers as in Fig. 3.1 (right) which shows the azimuthal correlation for $\gamma_{\text {rich }}$ sample triggers and $\pi^{0}$ triggers for the most peripheral and central bins. The $\gamma_{\text {rich }}$ sample has lower near-side yield than $\pi^{0}$ but not zero. The non-zero near-side yield for the $\gamma_{\text {rich }}$ sample is expected due to the remaining contributions of the widely separated photons from other sources. The shower shape analysis is only effective for the two close $\gamma$ showers.

The purity of using the shower shape analysis in $\pi^{0}$ identification is verified by comparing to previous measurements of azimuthal correlations between charged hadrons $(c h-c h)$ [21]. Figure 3.2 shows the $z_{T}$ dependence of the associated hadron yield normalized per $\pi^{0}$ trigger $D\left(z_{T}\right)$, where $z_{T}=p_{T}^{\text {assoc }} / p_{T}^{\text {trig }}$ [22], for the near-side and awayside compared to the per charged hadron trigger [21]. The near-side yield as in Fig. 3.2 (left) shows no significant difference between $p+p, d+\mathrm{Au}$, and $\mathrm{Au}+\mathrm{Au}$ indicating invacuum fragmentation even in heavy ion collisions, which 


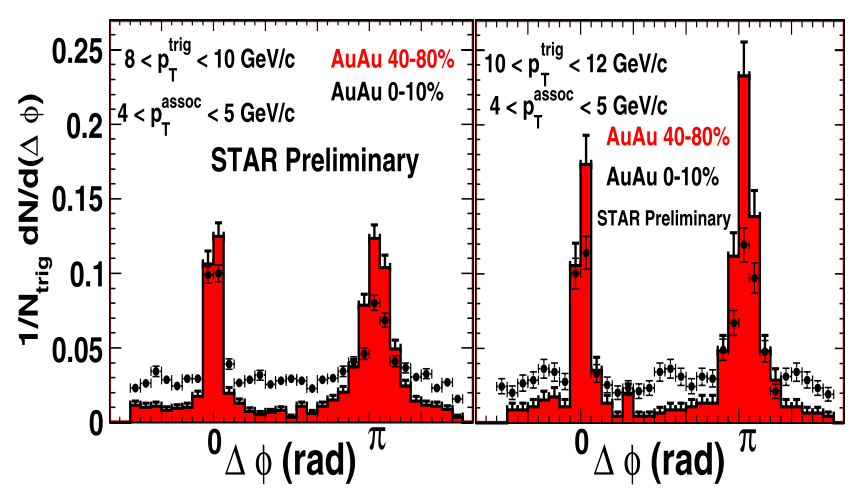

Fig. 3.1 Left: Azimuthal correlation histograms of high $p_{T}^{\text {trig }}$ inclusive photons with associated hadrons for $40-80 \%$ and $0-10 \% \mathrm{Au}+\mathrm{Au}$ collisions. Right: Azimuthal correlation histograms of high $p_{T}^{\text {trig }} \gamma_{\text {rich }}$

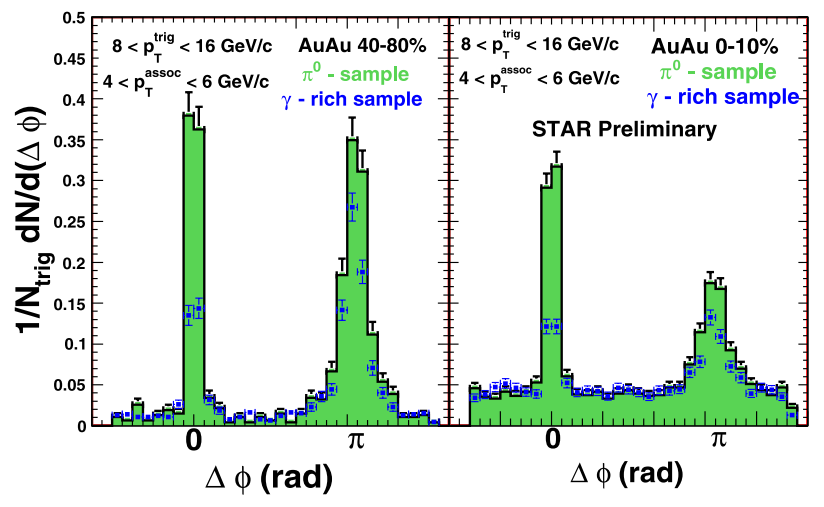

sample and $\pi^{0}$-sample with associated hadrons for $40-80 \%$ and 0 $10 \% \mathrm{Au}+\mathrm{Au}$ collisions
Fig. 3.2 $z_{T}$ dependence of $\pi^{0}-c h$ and $\operatorname{ch}-\operatorname{ch}[21]$ near-side (left panel) and away-side (right panel) associated particle yields

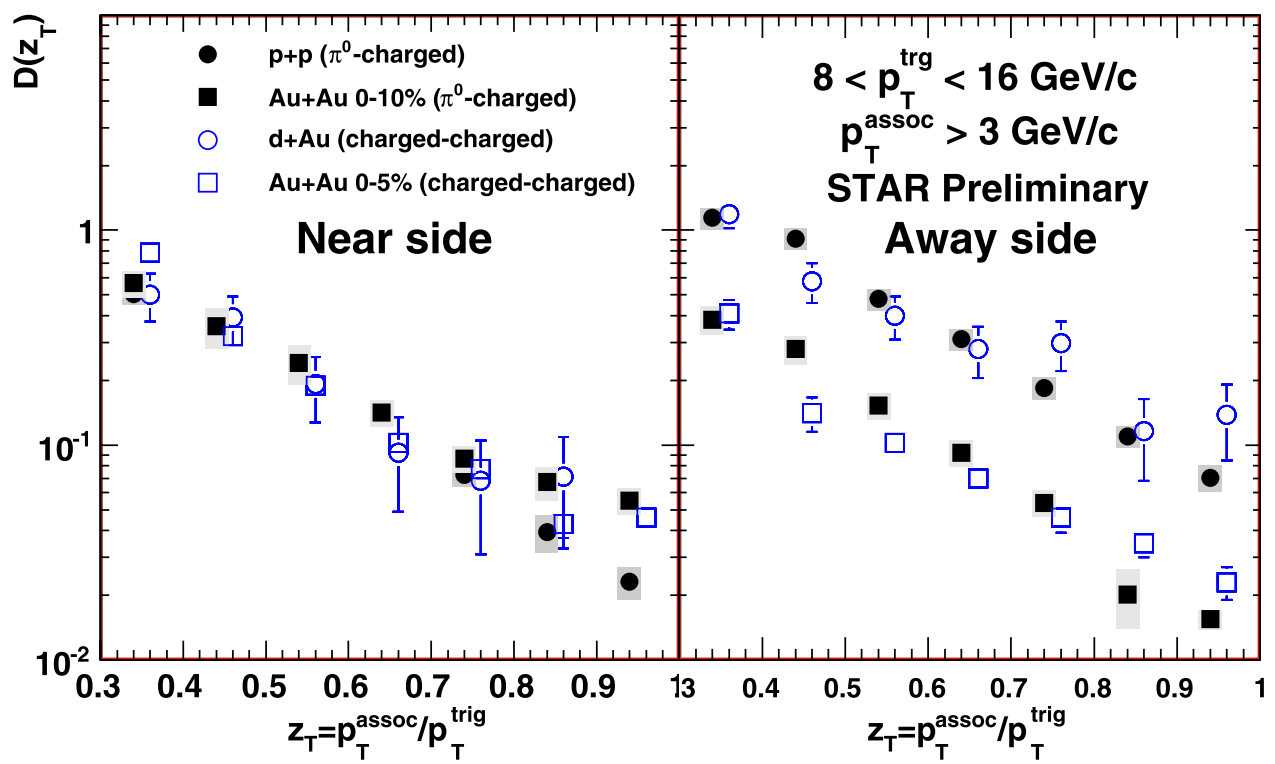

reveals the surface bias as generated in several model calculations [23-26]. However the medium effect is clearly seen in the away-side in Fig. 3.2 (right) where the per trigger yield in $\mathrm{Au}+\mathrm{Au}$ is significantly suppressed compared to $p+p$ and $d+\mathrm{Au}$. The general agreement between the results from this analysis $\left(\pi^{0}-c h\right)$ and the previous analysis $(c h-c h)$ is clearly seen in both panels of Fig. 3.2 which indicates the purity of the $\pi^{0}$ sample and therefore the effectiveness of shower shape analysis in $\pi^{0}$ identifications.

The away-side associated yields per trigger photon for direct $\gamma$-charged hadron correlations are extracted using Eq. 2.1. Figure 3.3 (left) shows the $z_{T}$ dependence of the trigger-normalized fragmentation function for $\pi^{0}$-charged correlations $\left(\pi^{0}-c h\right)$ compared to measurements with direct $\gamma$-charged correlations $(\gamma-c h)$. The away-side yield per trigger of direct $\gamma$ is smaller than with $\pi^{0}$ trigger at the same centrality class. This difference is due to the fact that the $\pi^{0}$ originates from higher initial parton energy and therefore supports the energy loss at the parton level before fragmentation as it was previously reported [27].

In order to quantify the away-side suppression, we calculate the quantity $I_{C P}$, which is defined as the ratio of the integrated yield of the away-side associated particles per trigger particle in $\mathrm{Au}+\mathrm{Au}$ central $0-10 \%$ of the geometrical cross section; relative to $\mathrm{Au}+\mathrm{Au}$ peripheral 40-80\% of the geometrical cross section collisions. Figure 3.3 (right) shows the $I_{C P}$ for $\pi^{0}$ triggers and for direct $\gamma$ triggers as a function of $z_{T}$. The ratio would be unity if there were no medium effects on the parton fragmentation; indeed the ratio deviates from unity by a factor of $\sim 2.5$. The ratio for the $\pi^{0}$ trigger is approximately independent of $z_{T}$ for the shown range in agreement with the previous results from $(c h-c h)$ measurements [21]. Within the current systematic uncertainty the $I_{C P}$ of direct $\gamma$ and $\pi^{0}$ are similar. 

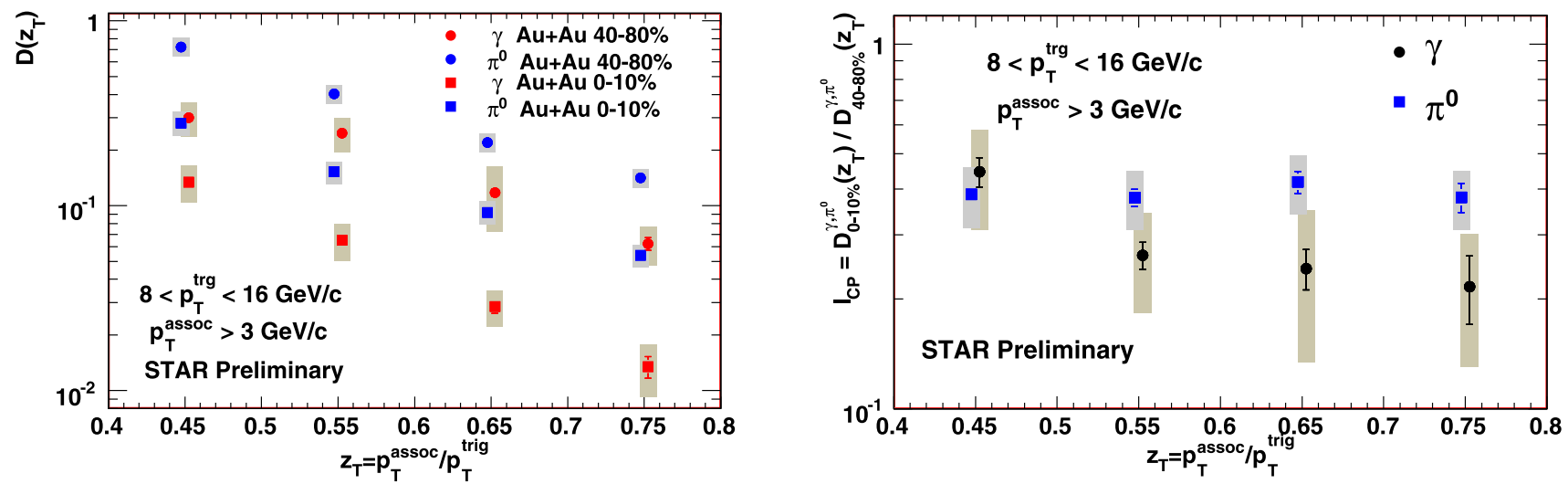

Fig. 3.3 (Left) $z_{T}$ dependence of $\pi^{0}$ triggers and direct $\gamma$ triggers associated particle yields for $40-80 \%$ and $0-10 \%$ Au + Au collisions. $($ Right $)$ $z_{T}$ dependence of $I_{C P}$ for direct $\gamma$ triggers and $\pi^{0}$ triggers (see text). Boxes show the systematic uncertainties

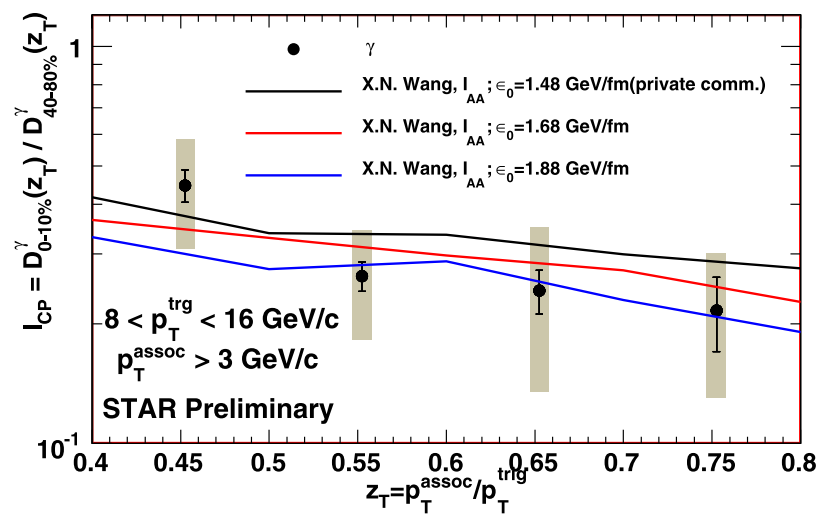

Fig. 3.4 $z_{T}$ dependence of $I_{C P}$ for direct $\gamma$ triggers associated particle yields compared with theoretical calculations (left) $I_{A A}$ of 0 $10 \% \mathrm{Au}+\mathrm{Au}$ collisions (Only Annihilation and Compton processes

Suppression ratios with respect to the $p+p$ reference, $I_{A A}$, have been reported earlier [28]. The values of $I_{A A}$ are smaller than for $I_{C P}$, indicating finite suppression in the peripheral $40-80 \%$ data, but the statistical uncertainties are large due to the small $\gamma / \pi^{0}$ ratio in $p+p$ as previously reported [29]. Although the value of $I_{A A}$ is found to be similar to the values observed for di-hadron correlations and for single-particle suppression $R_{A A}$.

A comparison of $I_{C P}$ of direct $\gamma$ with two theoretical model calculations of $I_{A A}$ of direct $\gamma$ is shown in Fig. 3.4. The $I_{C P}$ values agree well with the theoretical predictions within the current uncertainties. Figure 3.4 (left) indicates the need for more reduction in the systematic and statistical uncertainties in order to distinguish between different color charge densities.

\section{Summary and outlook}

In summary, a first measurement of fragment distributions for jets with a controlled energy via $\gamma$-jet in $\mathrm{Au}+\mathrm{Au}$ col-

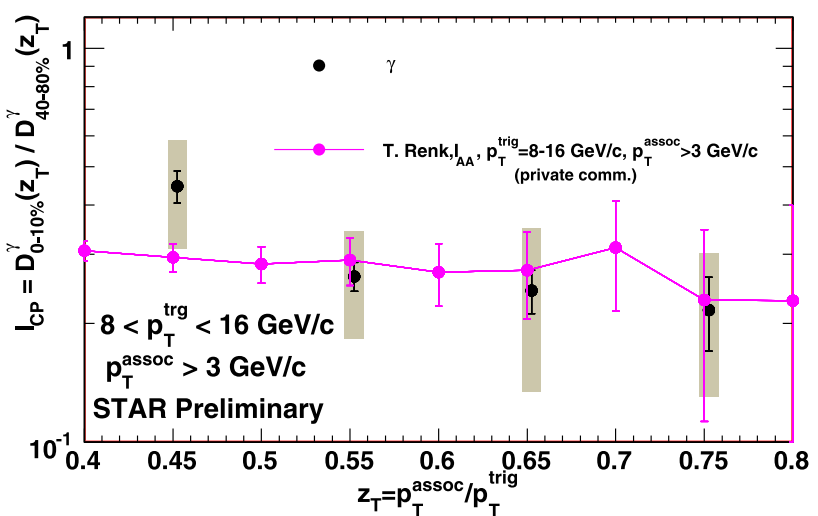

to NLO are considered) with three different initial gluon density where $7<p_{T}^{\text {trig }}<9 \mathrm{GeV} / c$ and $p_{T}^{\text {assoc }}>5 \mathrm{GeV} / c$, (right) $I_{A A}$ where $8<$ $p_{T}^{\text {trig }}<16 \mathrm{GeV} / c$ and $p_{T}^{\text {assoc }}>3 \mathrm{GeV} / c$

lisions has been performed by the STAR experiment. The STAR detector is unique to perform such correlation measurements due to the full coverage in azimuth. Within the current uncertainty the recoil suppression ratio $I_{C P}$ of direct $\gamma$ and $\pi^{0}$ are similar. A full analysis of the systematic uncertainties is under way and may lead to a reduction of the total uncertainty. Future RHIC runs will provide larger data samples to further reduce the uncertainties and extend the $z_{T}$ range.

\section{References}

1. I. Arsene et al., Nucl. Phys. A 757, 1 (2005)

2. B.B. Back et al., Nucl. Phys. A 757, 28 (2005)

3. J. Adams et al., Nucl. Phys. A 757, 102 (2005)

4. K. Adcox et al., Nucl. Phys. A 757, 184 (2005)

5. U.W. Heinz, P.F. Kolb, Nucl. Phys. A 702, 269 (2002)

6. D. Teaney, Phys. Rev. C 68, 034913 (2003)

7. P.K. Kovtun, D.T. Son, A.O. Starinets, Phys. Rev. Lett. 94, 111601 (2005) 
8. G. Policastro, D.T. Son, A.O. Starinets, Phys. Rev. Lett. 87, 081601 (2001)

9. X.N. Wang, M. Gyulassy, Phys. Rev. Lett. 68, 1480 (1992)

10. S. Adler et al., Phys. Rev. Lett. 91, 072303 (2003)

11. J. Adams et al., Phys. Rev. Lett. 91, 072304 (2003)

12. A. Majumder, J. Phys. G 34, S377-S388 (2007) and references therein

13. B.I. Abelev et al., Phys. Rev. Lett. 98, 192301 (2007)

14. B.I. Abelev et al., Phys. Rev. Lett. B 655, 104 (2007)

15. B.I. Abelev et al., Phys. Rev. Lett. 97, 152301 (2006)

16. P. Fachini (STAR Collaboration), in QM08 Proceedings, arXiv: 0808.3110

17. E. Eskola, H. Honkanen, C. Salgado, U. Wiedemann, Nucl. Phys. A 747, 511 (2005)

18. H. Zhang, J. Owens, E. Wang, X.N. Wang, Phys. Rev. Lett. 98, 212301 (2007)
19. X.N. Wang, Z. Huang, I. Sarcevic, Phys. Rev. Lett. 77 (1996)

20. K. Filimonov, Acta Phys. Hung. A 25, 363-370 (2006)

21. J. Adams et al., Phys. Rev. Lett. 97, 162301 (2006)

22. X.N. Wang, Phys. Lett. B 595, 165 (2004)

23. A. Drees, H. Feng, J. Jia, Phys. Rev. C 71, 034909 (2005)

24. A. Dainese, C. Loizides, G. Paic, Eur. Phys. J. C 38, 461 (2005)

25. K.J. Eskola et al., Nucl. Phys. A 747, 511 (2005)

26. B. Muller, Phys. Rev. C 67, 061901 (2003)

27. O. Catu (STAR Collaboration), in QM08 Proceedings

28. A.M. Hamed (STAR Collaboration), in QM08 Proceedings, arXiv:0806.2190

29. S. Chattopadhyay (STAR Collaboration), J. Phys. G: Nucl. Part. Phys. 34, S985-S988 (2007) 